\title{
Intestinal Gel Dosage Form
}

National Cancer Institute

\section{Source}

National Cancer Institute. Intestinal Gel Dosage Form. NCI Thesaurus. Code C149598.

Semi-solid preparation consisting of a gel intended for intestinal use. 